\title{
Zur Kenntnis der Doppelnitrate der seltenen Erden.
}

1. Mitteilung.

Über die Doppelnitrate der seltenen Erden mit den Alkalielementen. Von

G. JANTSCH und S. WIGDOROW.

Von den seltenen Erden sind namentlich die Elemente, die zur Gruppe der Ceriterden gehören, zur Bildung von Doppelnitraten befähigt. Es sind dies die Elemente: Lanthan, Cer, Praseodym, Neodym und Samarium. Von den Terbinerden sind nur beim Gado. linium Doppelnitrate bekannt. Die Elemente, die die Gruppe der Yttererden umfassen, scheinen nicht mehr die Fähigkeit zu besitzen, Doppelnitrate $\mathrm{zu}$ bilden. Während das Lanthan sehr stabile und leicht darstellbare Doppelnitrate bildet, sind die der folgenden Elemente weniger stabil. Die Doppelnitrate des Gadoliniums sind die am schwersten darstellbaren und die unstabilsten. Es scheint somit die Fähigkeit zur Bildung von Doppelnitraten bei den seltenen Erden mit dem Anwachsen des Atomgewichtes des seltenen Erdelementes geringer zu werden.

Die seltenen Erdelemente bilden Doppelnitrate mit den Alkalielementen, A mmonium, einigen zweiwertigen Elementen der Magnesiumreihe und auch, wie A. KOLB ${ }^{1}$ gefunden hat, mit organischen Basen. Während in den meisten Fällen bei den Doppelnitraten mit den Alkalielementen zwei Moleküle Alkalinitrat mit einem Molekül seltenen Erdnitrats vereinigt sind, entsprechen sämtliche Doppelnitrate mit den zweiwertigen Elementen der allgemeinen Formel:

$$
\left[\mathrm{Me} \mathrm{e}^{\mathrm{II}}\left(\mathrm{NO}_{3}\right)_{6}\right]_{2} \cdot \mathrm{Me}_{3}^{\mathrm{II}} \cdot 24 \mathrm{H}_{2} \mathrm{O} .
$$

Bis jetzt sind folgende Doppelnitrate der seltenen Erdelemente mit den Alkalielementen und mit Ammonium bekannt:

1 A. Kous, Z. anorg. Chem. 60 (1908), 123. 


$\begin{array}{ll}{\left[\mathrm{La}\left(\mathrm{NO}_{3}\right)_{5}\right]\left(\mathrm{NH}_{4}\right)_{2} \cdot 4 \mathrm{H}_{2} \mathrm{O}^{3}} & {\left[\mathrm{Ce}\left(\mathrm{NO}_{3}\right)_{5}\right] \mathrm{K}_{2} \cdot 2 \mathrm{H}_{2} \mathrm{O}^{7}} \\ {\left[\mathrm{Ce}\left(\mathrm{NO}_{3}\right)_{5}\right]\left(\mathrm{NH}_{4}\right)_{2} \cdot 4 \mathrm{H}_{2} \mathrm{O}^{2}} & {\left[\mathrm{P}\left(\mathrm{NO}_{3}\right)_{5}\right] \mathrm{Na}_{2} \cdot \mathrm{H}_{2} \mathrm{O}^{8}} \\ \left.\left[\mathrm{P}\left(\mathrm{NO}_{3}\right)_{5}\right] \mathrm{NH}_{4}\right)_{2} \cdot 4 \mathrm{H}_{2} \mathrm{O}^{3} & {\left[\mathrm{Nd}\left(\mathrm{NO}_{3}\right)_{5}\right] \mathrm{Na}_{2} \cdot \mathrm{H}_{2} \mathrm{O}^{9}} \\ {\left[\mathrm{Nd}\left(\mathrm{NO}_{3}\right)_{5}\right]\left(\mathrm{NH}_{4}\right)_{2} \cdot 4 \mathrm{H}_{2} \mathrm{O}^{4}} & \\ {\left[\mathrm{Gd}\left(\mathrm{NO}_{3}\right)_{5}\left(\mathrm{NH}_{4}\right)_{2} \cdot \mathrm{xH}_{2} \mathrm{O}^{5}\right.} & \\ 2 \mathrm{Ce}\left(\mathrm{NO}_{3}\right)_{3} \cdot 3 \mathrm{NH}_{4} \cdot \mathrm{NO}_{3} \cdot 12 \mathrm{H}_{2} \mathrm{O}^{6} & \end{array}$

In letzter Zeit hat G. WrrodBow ${ }^{10}$ die nachstehenden Doppelnitrate der Ceriterden mit den Alkalien beschrieben:

$\begin{array}{ll}3 \mathrm{LaO} . \mathrm{N}_{2} \mathrm{O}_{5} \cdot 2 \mathrm{Na}_{2} \mathrm{O} \cdot \mathrm{N}_{2} \mathrm{O}_{5} \cdot 3 \mathrm{H}_{2} \mathrm{O} & 3 \mathrm{CeO} \cdot \mathrm{N}_{2} \mathrm{O}_{5} \cdot 2 \mathrm{Rb}_{2} \mathrm{O} \cdot \mathrm{N}_{2} \mathrm{O}_{5} \cdot 8 \mathrm{H}_{2} \mathrm{O} \\ 3 \mathrm{LaO} . \mathrm{N}_{2} \mathrm{O}_{5} \cdot 2 \mathrm{~K}_{2} \mathrm{O} \cdot \mathrm{N}_{2} \mathrm{O}_{5} \cdot 4 \cdot 5 \mathrm{H}_{2} \mathrm{O} & 3 \mathrm{CeO} \cdot \mathrm{N}_{2} \mathrm{O}_{5} \cdot 2 \mathrm{Cs}_{2} \mathrm{O} \cdot \mathrm{N}_{2} \mathrm{O}_{5} \cdot 5 \mathrm{H}_{2} \mathrm{O} \\ 3 \mathrm{LaO} . \mathrm{N}_{2} \mathrm{O}_{5} \cdot 2 \mathrm{Rb}_{2} \mathrm{O} \cdot \mathrm{N}_{2} \mathrm{O}_{5} \cdot 8 \mathrm{H}_{2} \mathrm{O} & 3 \mathrm{DiO} \cdot \mathrm{N}_{2} \mathrm{O}_{5} \cdot 2 \mathrm{Rb}_{2} \mathrm{O} \cdot \mathrm{N}_{2} \mathrm{O}_{5} \cdot 8 \mathrm{H}_{2} \mathrm{O} \\ 3 \mathrm{LaO} . \mathrm{N}_{2} \mathrm{O}_{5} \cdot 2 \mathrm{Cs}_{2} \mathrm{O} \cdot \mathrm{N}_{2} \mathrm{O}_{5} \cdot 5 \mathrm{H}_{2} \mathrm{O} & 5 \mathrm{DiO} \cdot \mathrm{N}_{2} \mathrm{O}_{5} \cdot 6 \mathrm{NH}_{4} \mathrm{NO}_{3} \cdot{ }^{11}\end{array}$

Nach den heute geltenden Anschaungen, nach welchen die seltenen Erdelemente in ihren stabilen Verbindungsformen als dreiwertig aufgefalst werden, müssen diese Doppelnitrate folgendermalsen formuliert werden:

$$
\begin{array}{ll}
{\left[\mathrm{La}\left(\mathrm{NO}_{3}\right)_{5}\right] \mathrm{Na}_{2} \cdot 11_{2} \mathrm{H}_{2} \mathrm{O}} & {\left[\mathrm{Ce}\left(\mathrm{NO}_{3}\right)_{5}\right] \mathrm{Rb}_{2} \cdot 4 \mathrm{H}_{2} \mathrm{O}} \\
{\left[\mathrm{La}\left(\mathrm{NO}_{3}\right)_{5}\right] \mathrm{K}_{2} \cdot 2{ }^{1}{ }_{4} \mathrm{H}_{2} \mathrm{O}} & {\left[\mathrm{Ce}\left(\mathrm{NO}_{3}\right)_{5} \mathrm{Cs}_{2} \cdot 2{ }_{2} \mathrm{H}_{2} \mathrm{O}\right.} \\
{\left[\mathrm{La}\left(\mathrm{NO}_{3}\right)_{5}\right] \mathrm{Rb}_{2} \cdot 4 \mathrm{H}_{2} \mathrm{O}} & {\left[\mathrm{Di}\left(\mathrm{NO}_{3}\right)_{5}\right] \mathrm{Rb}_{2} \cdot 4 \mathrm{H}_{2} \mathrm{O}} \\
{\left[\mathrm{La}\left(\mathrm{NO}_{3}\right)_{5}\right] \mathrm{Cs}_{2} \cdot 2{ }^{1}{ }_{2} \mathrm{H}_{2} \mathrm{O}} &
\end{array}
$$

Durch kristallographische Messungen hat G. WyrodBow festgestellt, dafs von diesen Salzen nur die Rubidiumdoppelnitrate mit den Ammondoppelnitraten isomorph sind.

1 Descloirceaux, Jahresber. 1858, 132. 135. - C. Marianac, Ann. chim. phys. [4] 30 (1873), 67. - C. Auer von Welsbach, M. 6 (1885), 477. - Dufet, Bull. Soc. franc. Minéral 11 (1888), 143. 215; Z. f. Kristall. 18 (1891), 444. -E. H. KraUs, Z. f. Kristall. 34 (1901), 307.

${ }^{2}$ C. Marianac, Ann. chim. phys. [4] 30 (1873), 67. - Fook, Z. f. Kristall. 22 (1894), 37. - H. Wolf, Z. anorg. Chem. 45 (1905), 89.

${ }^{3}$ C. Auer von Welsbach, $M .6$ (1885), 477. - C. von Scheele, Z. anorg. Chem. 18 (1898), 352.

4 C. Auer von Welsbach, M. 6 (1885), 477.

5 Abeggs Handbuch der anorg. Chemie III 1, (1906), S. 307.

${ }^{6}$ M. Holzmann, Journ. prakt. Chem. 84 (1861), 76.

${ }^{7}$ L. Th. LaNae, Journ. prakt. Chem. 82 (1861), 129.

8 C. Auer von Wellasbach, M. 6 (1885), 477.

9 C. Auer von Wellsbach, M. 6 (1885), 477.

10 G. Wrnodbow, Bull. Soc. frane. Minéral 29 (1907), 324; Ebenda 30 (1908), 299.

1. G. W чrodвow, Bull. Soc. franc. Minéral 32 (1909), 365. 
Wir haben die von G. Wrroubow beschriebenen Doppelnitrate nochmals dargestellt und gefunden, dals den Natrium-, den Kaliumund den Cäsiumsalzen ein anderer Wassergehalt zukommt, als ihn G. Wrroubow angegeben hat. Bei den Rubidiumdoppelnitraten konnten wir die Angaben G. WyrodBows bestätigen. Ferner haben wir die Rubidiumdoppelnitrate des Praseodyms und Neodyms und die Doppelnitrate des Lanthans und des Cers mit dem einwertigen Thallium dargestellt. Wir bemühten uns, auch die Lithiumdoppelnitrate mit den seltenen Erden zu erhalten. Wir hatten iedoch damit keinen Erfolg, was wohl seinen Grund in der überaus grolsen Löslichkeit dieser Salze hat.

Die Rubidiumdoppelnitrate des Praseodyms und Neodyms kristallisieren, wie zu erwarten war, mit 4 Molekülen Wasser. Den gleichen Wassergehalt besitzen auch die Thalliumdoppelnitrate.

Den Natrium-, Kalium- und Cäsiumdoppelnitraten kommen nach unseren Untersuchungen folgende Formeln zu:

$$
\begin{array}{ll}
{\left[\mathrm{La}\left(\mathrm{NO}_{3}\right)_{5}\right] \mathrm{Na}_{2} \cdot \mathrm{H}_{2} \mathrm{O}} & {\left[\mathrm{Ce}\left(\mathrm{NO}_{3}\right)_{5}\right] \mathrm{Na}_{2} \cdot \mathrm{H}_{2} \mathrm{O}} \\
{\left[\mathrm{La}\left(\mathrm{NO}_{3}\right)_{5}\right] \mathrm{K}_{2} \cdot 2 \mathrm{H}_{2} \mathrm{O}} & \\
{\left[\mathrm{La}\left(\mathrm{NO}_{3}\right)_{5}\right] \mathrm{Cs}_{2} \cdot 2 \mathrm{H}_{2} \mathrm{O}} &
\end{array}
$$

Die Rubidium- und die Thalliumdoppelnitrate besitzen im Gegensatze zu denen der anderen Alkalielemente die Eigenschaft, bei einer bestimmten Temperatur ziemlich scharf in ihrem Kristallwasser zu schmelzen. Diese Schmelzpunkte sinken, wie folgende kleine Tabelle zeigt, bei den einzelnen Salzen mit dem Anwachsen des Atomgewichtes des seltenen Erdelementes:

S. P.

$\left[\mathrm{La}\left(\mathrm{NO}_{3}\right)_{5}\right] \mathrm{Rb}_{2} \cdot 4 \mathrm{H}_{2} \mathrm{O} \quad 86^{\circ}$

$\left.{ }^{r} \mathrm{Ce}\left(\mathrm{NO}_{3}\right)_{5}\right] \mathrm{Rb}_{2} \cdot 4 \mathrm{H}_{2} \mathrm{O} \quad 70^{\circ}$

$\left[\mathrm{P}\left(\mathrm{NO}_{3}\right)_{5}\right] \mathrm{Rb}_{2} \cdot 4 \mathrm{H}_{2} \mathrm{O} \quad 63.5^{\circ}$

$\left[\mathrm{Nd}\left(\mathrm{NO}_{3}\right)_{5}\right] \mathrm{Rb}_{2} .4 \mathrm{H}_{2} \mathrm{O} \quad 47^{\circ}$
S. P.

$\left[\mathrm{La}\left(\mathrm{NO}_{3}\right)_{5}\right] \mathrm{Tl}_{2} .4 \mathrm{H}_{2} \mathrm{O} \quad 72^{0}$

$\left[\mathrm{Ce}\left(\mathrm{NO}_{3}\right)_{5}\right] \mathrm{Tl}_{2} \cdot 4 \mathrm{H}_{2} \mathrm{O} \quad 64.5^{0}$

Ferner haben wir die Dichten aller von uns untersuchten Doppelnitrate bei $0^{\circ}$ bestimmt und aus ihnen die Molekularvolumina für die einzelnen Salze berechnet. Die erhaltenen Werte sind in der folgenden Übersicht zusammengestellt:

$\begin{array}{lcc} & d_{4^{0}=}^{0^{0}} & \text { Molekularvolumen } \\ \left.\left[\mathrm{LaNO}_{3}\right)_{5}\right] \mathrm{Na}_{2} \cdot \mathrm{H}_{2} \mathrm{O} & 2.63 & 195.08 \\ {\left[\mathrm{Ce}\left(\mathrm{NO}_{3}\right)_{5}\right] \mathrm{Na}_{2} \cdot \mathrm{H}_{2} \mathrm{O}} & 2.65 & 194.00\end{array}$




$\begin{array}{llc} & d_{4,}^{00}= & \text { Molekularrolumen } \\ {\left[\mathrm{La}\left(\mathrm{NO}_{3}\right)_{5}\right] \mathrm{K}_{2} \cdot 2 \mathrm{H}_{2} \mathrm{O}} & 2.54 & 221.76 \\ {\left[\mathrm{La}\left(\mathrm{NO}_{3}\right)_{5}\right] \mathrm{Cs}_{2} \cdot 2 \mathrm{H}_{2} \mathrm{O}} & 2.827 & 265.5 \\ {\left[\mathrm{La}\left(\mathrm{NO}_{3}\right)_{5}\right] \mathrm{Rb}_{2} \cdot 4 \mathrm{H}_{2} \mathrm{O}} & 2.497 & 277.1 \\ {\left[\mathrm{La}\left(\mathrm{NO}_{3}\right)_{5}\right] \mathrm{T}_{2} \cdot 4 \mathrm{H}_{2} \mathrm{O}} & 3.318 & 280.0 \\ {\left[\mathrm{Ce}\left(\mathrm{NO}_{3}\right)_{5}\right] \mathrm{Rb}_{2} \cdot 4 \mathrm{H}_{2} \mathrm{O}} & 2.497 & 277.6 \\ {\left[\mathrm{Ce}\left(\mathrm{NO}_{3}\right)_{5}\right] \mathrm{Tl}_{2} \cdot 4 \mathrm{H}_{2} \mathrm{O}} & 3.326 & 279.7 \\ {\left[\mathrm{P}\left(\mathrm{NO}_{3}\right)_{5}\right] \mathrm{Rb}_{2} \cdot 4 \mathrm{H}_{2} \mathrm{O}} & 2.50 & 277.4 \\ {\left[\mathrm{Nd}\left(\mathrm{NO}_{3}\right)_{5}\right] \mathrm{Rb}_{2} \cdot 4 \mathrm{H}_{3} \mathrm{O}} & 2.56 & 272.3\end{array}$

Da bekanntlich isomorphe Verbindungen in der Regel ein ziemlich ähnliches Molekularvolumen besitzen, so glauben wir, wie aus dieser Zusammenstellung hervorgeht, annehmen zu können, dafs die Thalliumdoppelnitrate mit den Rubidium- und somit auch mit den Ammoniumdoppelnitraten isomorph sind.

Aufser dem Lanthan-Rubidiumnitrat der Formel $\left[\mathrm{La}\left(\mathrm{NO}_{3}\right)_{5}\right] \mathrm{Rb} b_{2}$. $4 \mathrm{H}_{2} \mathrm{O}$, gelang es uns, auch ein saures $\mathrm{Salz}\left[\mathrm{La}\left(\mathrm{NO}_{3}\right)_{4}\right] \mathrm{Rb} . \mathrm{HNO}_{3}$. $6 \mathrm{H}_{2} \mathrm{O}$ darzustellen. Beim dreiwertigen Cer konnten wir jedoch die Bildung eines ähnlich zusammengesetzten Doppelnitrates nicht beobachten.

\section{Experimenteller 'Teil.}

\section{Lanthan-Natriumnitrat, $\left[\mathrm{La}\left(\mathrm{NO}_{3}\right)_{5}\right] \mathrm{Na}_{2} \cdot \mathrm{H}_{2} \mathrm{O}$.}

5 g Lanthanoxyd und $3.27 \mathrm{~g}$ Natriumkarbonat werden mit Wasser angeteigt und in konzentrierter Salpetersäure unter Vermeidung eines grölseren Überschusses gelöst. Die Mengenverhältnisse sind dabei so gewählt, dafs auf 1 Molekül $\mathrm{La}_{2} \mathrm{O}_{3} 2$ Moleküle $\mathrm{Na}_{2} \mathrm{CO}_{3}$ kommen. Sobald vollständige Lösung eingetreten ist, dampft man bis zum Erscheinen einer Kristallhaut ein, worauf nach dem Erkalten das Doppelnitrat auszukristallisieren beginnt. Durch scharfes Absaugen werden die Kristalle von der Mutterlauge getrennt und nachher über Kaliumbydroxyd getrockuet. Nach dem Eindampfen kann aus der Mutterlauge eine zweite Kristallfraktion gewonnen werden. Die Ausbeute beträgt $11 \mathrm{~g}$.

Das Lanthan-Natriumnitrat besteht aus weifsen, feinnadeligen Kristallen, die sich in Wasser langsam, jedoch in grofser Menge mit nentraler Reaktion lösen. Das Salz aus Wasser umzukristallisieren, gelingt nicht. Aus verdünnter Salpetersäure kann es jedoch, wenn man mit sehr konzentrierten Lösungen arbeitet, umkristallisiert 
werden. Durch Erhitzen bis auf $150^{\circ}$ gelingt es noch nicht, sämtliches Wasser aus dem Salze zu entfernen.

$0.288 \mathrm{~g}$ Subst.: $0.0911 \mathrm{~g} \mathrm{La}_{2} \mathrm{O}_{3} ; 0.7734 \mathrm{~g}$ Subst.: $0.2166 \mathrm{~g} \mathrm{Na}_{2} \mathrm{SO}_{4}$; $0.0624 \mathrm{~g}$ Subst.: $15 \mathrm{~cm}$ NO bei $732.2 \mathrm{~mm}, 18^{\circ}$.

Für $\left[\mathrm{La}\left(\mathrm{NO}_{3 / 5}\right)\right] \mathrm{Na}_{2} \cdot \mathrm{H}_{2} \mathrm{O}$ ber. $\mathrm{La}_{2} \mathrm{O}_{3} 31.77 \quad \mathrm{Na} 8.96 \quad$ NO 29.24 gef. $\quad \mathrm{La}_{2} \mathrm{O}_{3} 31.63 \quad \mathrm{Na} 9.06 \quad \mathrm{NO} 29.16$

Die Bestimmung der Dichte, in o-Chlortoluol bei $0^{0}$ nach der Pyknometermethode ausgeführt, ergab $d_{4^{0}}^{00}=2.63$. Das Molekularvolumen ist daher 195.08 .

\section{Lanthan-Kaliumnitrat $\left[\mathrm{La}\left(\mathrm{NO}_{3}\right)_{5}\right] \mathrm{K}_{2} \cdot 2 \mathrm{H}_{2} \mathrm{O}$.}

Die Darstellung dieses Salzes ist der des Natriumsalzes ganz analog. Aus $3 \mathrm{~g}$ Lanthanoxyd werden $13 \mathrm{~g}$ Doppelnitrat erhalten. Auch hier gelingt es nicht, das Salz aus Wasser umzukristallisieren.

Das Lanthan-Kaliumnitrat kristallisiert in kleinen weifsen, glänzenden Kristallen, die sehr hygroskopisch sind und sich in Wasser mit neutraler Reaktion lösen. Beim Erhitzen auf $60^{\circ}$ verliert das Salz sämtliches Kristallwasser.

0.3812 g Subst.: $0.1109 \mathrm{~g} \mathrm{La}_{2} \mathrm{O}_{3} ; 0.0235 \mathrm{~g}$ Subst.: $5.2 \mathrm{ccm}$ NO bei $725 \mathrm{~mm}, 19^{\circ} ; 0.2756 \mathrm{~g}$ Subst.: $0.0866 \mathrm{~g} \mathrm{~K}_{2} \mathrm{SO}_{4} ; 0.4477 \mathrm{~g}$ Subst. verlieren bei $60^{\circ} 0.0288 \mathrm{~g} \mathrm{H}_{2} \mathrm{O}$.

Für $\left[\mathrm{La}\left(\mathrm{NO}_{3}\right)_{5}\right] \mathrm{K}_{2} \cdot 2 \mathrm{H}_{2} \mathrm{O}$ ber. $\mathrm{La}_{2} \mathrm{O}_{3} 28.93 \mathrm{~K} 13.89 \mathrm{NO} 26.63 \mathrm{H}_{2} \mathrm{O} 6.39$ gef. $\mathrm{La}_{2} \mathrm{O}_{3} 29.09 \mathrm{~K} 14.10 \mathrm{NO} 26.40 \mathrm{H}_{2} \mathrm{O} 6.43$

Die Ermittelung der Dichte bei $0^{\circ}$ in o-Chlortoluol nach der Pyknometermethode ausgeführt, ergab $d_{40}^{00}=2.54$. Somit ist das Molekularvolumen 221.76.

\section{Saures Lanthan-Rubidiumnitrat $\left.\mathrm{La}\left(\mathrm{NO}_{3}\right)_{4}\right] \mathrm{Rb} . \mathrm{HNO}_{3} \cdot 6 \mathrm{H}_{2} \mathrm{O}$.}

Versucht man, anaiog den Darstellungsweisen der oben beschriebenen Verbindungen, das entsprechende Rubidiumsalz durch Kristallisation von salpetersauren Lösungen von 1 Molekül Lanthannitrat und 2 Molekülen Rubidiumnitrat darzustellen, so erhält man, entgegen den Erwartungen, nicht das Salz $\left[\mathrm{La}\left(\mathrm{NO}_{3}\right)_{5}\right] \mathrm{Rb}_{2} \cdot \mathrm{xH}_{2} \mathrm{O}$, sondern ein saures Salz der Formel $\left[\mathrm{La}\left(\mathrm{NO}_{3}\right)_{4}\right] \mathrm{Rb} . \mathrm{HNO}_{3} \cdot 6 \mathrm{H}_{2} \mathrm{O}$. Die Bildung dieser Verbindung wird wahrscheinlich durch die grolse Tendenz des Rubidiumnitrats Salpetersäure anzulagern bedingt. 
5 g Lanthanoxyd und $7.1 \mathrm{~g}$ Rubidiumkarbonat werden nach dem Verrühren mit wenig Wasser vorsichtig in Salpetersäure gelöst. Aus der am Wasserbade ziemlich stark konzentrierten Lösung kristallisiert nach dem Erkalten das Doppelnitrat in farblosen, tafeligen Kristallen aus, die dann behufs Reinigung aus konzentrierter Salpetersäure umkristallisiert werden können. Die Ausbeute beträgt hierbei $11.5 \mathrm{~g}$.

Das saure Lanthan-Rubidiumnitrat ist in Wasser, wie auch in Salpetersäure sehr leicht löslich. Seine wässerige Lösung zeigt stark saure Reaktion und verbraucht beim Titrieren mit Kalilauge genau die einem Molekül $\mathrm{HNO}_{3}$ entsprechende Menge $\mathrm{KOH}$. Beim Erhitzen auf $120^{\circ}$ entweichen aus dem Salze 5 Moleküle Wasser und 1 Molekül Salpetersäure, und es verbleibt ein Salz, dessen Zusammensetzung ziemlich genau auf die Formel $\left[\mathrm{La}\left(\mathrm{NO}_{3}\right)_{4}\right] \mathrm{Rb} . \mathrm{H}_{2} \mathrm{O}$ stimmt. Das letzte Molekül Wasser kann aus dem Salze auch beim Erhitzen bis auf $200^{\circ}$ nicht entfernt werden.

0.4519 g Subst: $0.1145 \mathrm{~g} \mathrm{La}_{2} \mathrm{O}_{3} ; 0.3333 \mathrm{~g}$ Subst: $0.0707 \mathrm{~g}$ $\mathrm{Rb}_{2} \mathrm{SO}_{4} ; 0.0544 \mathrm{~g}$ Subt.: $10.6 \mathrm{ccm}$ NO bei $727.5 \mathrm{~mm}, 19^{\circ} ; 0.4748 \mathrm{~g}$ Subst. verbrauchten $7.3 \mathrm{ccm} 1 / 10^{-n o r m}$. KOH; $0.4996 \mathrm{~g}$ Subst. verlieren bei $120^{\circ} 0.1196 \mathrm{~g}$.

Für $\left[\mathrm{La}\left(\mathrm{NO}_{3}\right)_{4}\right] \mathrm{Rb} . \mathrm{HNO}_{3} .6 \mathrm{H}_{2} \mathrm{O}$ ber. $\mathrm{La}_{2} \mathrm{O}_{3} 25.31 \mathrm{Rb} 13.27 \mathrm{HNO}_{3} 9.79$ NO $23.315 \mathrm{H}_{2} \mathrm{O} . \mathrm{HNO}_{3} 23.78$

Für $\left[\mathrm{La}\left(\mathrm{NO}_{3}\right)_{4}\right] \mathrm{Rb} . \mathrm{HNO}_{3} \cdot 6 \mathrm{H}_{2} \mathrm{O}$ gef. $\mathrm{La}_{2} \mathrm{O}_{3} 25.34 \mathrm{Rb} 13.57 \mathrm{HNO}_{3} 9.68$ NO $23.39 \quad 5 \mathrm{H}_{2} \mathrm{O} . \mathrm{HNO}_{3} 23.93$

Die Ermittelung der Dichte bei $0^{0}$ ergab $d_{4^{0}}^{0^{0}}=2.377$. Das Molekularvolumen ist daher 270.6.

Analyse des bei $120^{\circ}$ getrockneten Salzes:

$0.1728 \mathrm{~g}$ Subst.: $0.0585 \mathrm{~g} \mathrm{La}_{2} \mathrm{O}_{3} ; 0.0541 \mathrm{~g}$ Subst.: $11.2 \mathrm{ccm}$ NO bei $726 \mathrm{~mm}, 18.5^{0}$.

Für $\left[\mathrm{La}\left(\mathrm{NO}_{3}\right)_{4}\right] \mathrm{Rb} . \mathrm{H}_{2} \mathrm{O}$ berechnet $\mathrm{La}_{2} \mathrm{O}_{3} 33.23 \quad \mathrm{NO} 24.47$ gefunden $\mathrm{La}_{2} \mathrm{O}_{3} 3385$ NO 24.87

Lanthan-Rubidiumnitrat $\left[\mathrm{La}\left(\mathrm{NO}_{3}\right)_{5}\right] \mathrm{Rb}_{2} .4 \mathrm{H}_{2} \mathrm{O}$.

Das Lanthan-Rubidiumnitrat läIst sich leicht durch Erhöhung der Rubidiumnitratkonzentration darstellen. Man verfährt am besten so, dafs in der Lösung auf 1 Molekiil Lanthannitrat 4 Moleküle Rubidiumnitrat vorhanden sind. 
$3 \mathrm{~g}$ Lanthanoxyd und $8.5 \mathrm{~g}$ Rubidiumnitrat werden, nach dem Umrühren mit wenig Wasser unter Vermeidung eines grofsen Überschusses in Salpetersäure gelöst. Die Lösung wird am Wasserbade konzentriert, worauf das Salz beim Erkalten auskristallisiert. Nach kurzer Zeit wird die Kristallisation unterbrochen, ein gut ausgebildeter Kristall herausgenommen und das Ganze unter Erwärmen und Zugabe einiger Tropfen Wasser wieder gelöst. Nach dem Erkalten wird mit dem Kristall geimpft, worauf das Doppelnitrat in gut ausgebildeten, monoklinen, glänzenden Kristallen erhalten wird.

Dàs Lanthan-Rubidiumnitrat löst sich sehr leicht mit neutraler Reaktion in Wasser und schmilzt ziemlich scharf bei $86^{\circ}$ in seinem Kristallwasser. Durch andauerndes Erhitzen auf $60^{\circ}$ lälst sich alles Wasser aus dem Salze entfernen.

$0.4180 \mathrm{~g}$ Subst.: $0.0986 \mathrm{~g} \mathrm{La}_{2} \mathrm{O}_{3} ; 0.4180 \mathrm{~g}$ Subst.: $0.1628 \mathrm{~g}$ $\mathrm{Rb}_{2} \mathrm{SO}_{4} ; 0.0582 \mathrm{~g}$ Subst.: $10.8 \mathrm{ccm}$ NO bei $720 \mathrm{~mm}, 23^{\circ} ; 0.4992 \mathrm{~g}$ Subst. verloren bei $60^{\circ} 0.0551 \mathrm{~g} \mathrm{H}_{2} \mathrm{O}$.

Für[La( $\left.\left(\mathrm{NO}_{3}\right)_{5}\right] \mathrm{Rb}_{2} \cdot 4 \mathrm{H}_{2} \mathrm{O}$ ber. $\mathrm{La}_{2} \mathrm{O}_{3} 23.55 \mathrm{Rb} 24.69 \mathrm{NO} 21.68 \mathrm{H}_{2} \mathrm{O} 10.41$ gef. $\mathrm{La}_{2} \mathrm{O}_{3} 23.58 \mathrm{Rb} 24.93 \mathrm{NO} 21.76 \mathrm{H}_{2} \mathrm{O} 11.03$

Die Bestimmung der Dichte, bei $0^{\circ}$ in o-Chlortoluol nach der Pyknometermethode ausgeführt, ergab $d_{4^{\circ}}^{00}=2.497$. Somit ist das Molekularvolumen 277.1.

\section{Lanthan-Cäsiumnitrat $\left[\mathrm{La}\left(\mathrm{NO}_{3}\right)_{5}\right] \mathrm{Cs}_{2} \cdot 2 \mathrm{H}_{2} \mathrm{O}$.}

Die Darstellung des Lanthan-Cäsiumnitrats ist der des Kaliumsalzes vollkommen gleich. Auf $5 \mathrm{~g}$ Lanthanoxyd werden $12.56 \mathrm{~g}$ Cäsiumnitrat angewendet. Die Ausbeute beträgt dann 13.5 g Doppelnitrat. Um ein einheitliches Produkt zu erhalten, ist eine ganz bestimmte Konzentration erforderlich, die man erst nach einiger Übung erreicht.

Das Lanthan-Cäsiumnitrat stellt matte, kleine tafelige Kristalle dar. Das Salz aus Wasser umzukristallisieren gelingt nicht. Nur durch andauerndes Trocknen über Kaliumhydroxyd im Vakuum ist es möglich, die anhaftende Salpetersäure vollständig zu entfernen.

0.5080 g Subst.: $0.1102 \mathrm{~g} \mathrm{La}_{2} \mathrm{O}_{3} ; 0.0666 \mathrm{~g}$ Subst.: $11.1 \mathrm{ccm}$ NO bei $725 \mathrm{~mm}, 18^{0} ; 0.4445 \mathrm{~g}$ Subst.: $0.2138 \mathrm{~g} \mathrm{Cs}_{2} \mathrm{SO}_{4} ; 0.4662 \mathrm{~g}$ Subst.: $0.0228 \mathrm{~g}$ Verlust.

Für $\left[\mathrm{La}\left(\mathrm{NO}_{3}\right)_{5}\right] \mathrm{Cs}_{2} \cdot 2 \mathrm{H}_{2} \mathrm{O}$ ber. $\mathrm{La}_{2} \mathrm{O}_{3} 21.71$ Cs 35.38 NO $19.98 \mathrm{H}_{2} \mathrm{O} 4.79$ gef. $\mathrm{La}_{2} \mathrm{O}_{3} 21.70 \mathrm{Cs} 35.32 \mathrm{NO} 20.02 \mathrm{H}_{2} \mathrm{O} 4.89$ 
Die Ermittelung der Dichte bei $0^{0}$ ergah $d_{4{ }^{0}}^{00}=2.827$. Das Molekularvolumen ist daher 265.5 .

\section{Lanthan-Thallonitrat $\left[\mathrm{La}\left(\mathrm{NO}_{3}\right)_{5}\right] \mathrm{Tl}_{2} .4 \mathrm{H}_{2} \mathrm{O}$.}

Die Darstellung des Lanthan-Thallonitrats gelingt nur dann, wenn man mit einem Überschufs von Lantbannitrat in der Lösung arbeitet, weil sich sonst das schwerlösliche Thallonitrat ausscheidet.

$3 \mathrm{~g}$ Lanthanoxyd und $4.31 \mathrm{~g}$ Thalliumkarbonat (Verhältnis 1 Molekül $\mathrm{La}_{2} \mathrm{O}_{3}: 1$ Molekül $\mathrm{N}_{2} \mathrm{CO}_{3}$ ) werden in Salpetersäure gelöst und die Lösung am Wasserbade konzentriert. Sobald die nötige Konzentration erreicht ist, die auch hier erst nach einiger Übung gefunden wird, kristallisiert nach dem Erkalten das Lanthan-Thallonitrat in derben, weifsen, glänzenden Kristallen aus. Es ist ratsam, die Kristalle nicht zu lange mit der Mutterlauge in Berührung zu lassen, weil sonst das überschüssige Lanthannitrat auch auskristallisiert. Die Ausbeute beträgt $11 \mathrm{~g}$.

Das Lanthan-Thallonitrat ist sehr bygroskopisch und schmilzt bei $72^{\circ}$ in seinem Kristallwasser. Mit einer Spur Wasser versetzt, zersetzt es sich sofort unter Abscheidung von Thallonitrat. Die wässerige Lösung reagiert neutral.

$0.3140 \mathrm{~g}$ Subst.: $0.0552 \mathrm{~g} \mathrm{La}_{2} \mathrm{O}_{3} ; 0.2307 \mathrm{~g}$ Subst. verbrauchten $10.0 \mathrm{ccm} \mathrm{KMnO}_{4}$-Lösung. $1 \mathrm{ccm} \mathrm{KMnO}_{4}$-Lösung $=0.010153 \mathrm{~g} \mathrm{Tl}$. $0.0569 \mathrm{~g}$ Subst.: $7.8 \mathrm{ccm}$ NO bei $720 \mathrm{~mm}, 18.5^{\circ} ; 0.3720 \mathrm{~g}$ Subst. verloren bei $100^{\circ} 0.0302 \mathrm{~g}$.

Für $\left[\mathrm{La}\left(\mathrm{NO}_{3}\right)_{5}\right] \mathrm{Tl}_{2} .4 \mathrm{H}_{2} \mathrm{O}$ ber. $\mathrm{La}_{2} \mathrm{O}_{3} 17.54 \mathrm{Tl} 43.92 \mathrm{NO} 16.15 \mathrm{H}_{2} \mathrm{O} 7.75$ gef. $\mathrm{La}_{2} \mathrm{O}_{3} 17.58 \mathrm{Tl} 44.00 \mathrm{NO} 16.33 \mathrm{H}_{2} \mathrm{O} \& .11$

Die Dichtebestimmung ergab $d_{4^{0}}^{0^{0}}=3.318$. Das Molekularvolumen ist somit 280.0 .

Cero-Natriumnitrat $\left[\mathrm{Ce}\left(\mathrm{NO}_{3}\right)_{5}\right] \mathbf{N a}_{2} \cdot \mathrm{H}_{2} \mathbf{O}$.

Die Darstellung dieses Salzes gleicht der des entsprechenden Lanthandoppelnitrats. Wegen der oxydierenden Wirkung der Salpetersäure wird beim Konzentrieren der Lösung stets ein Teil des Cers zur vierwertigen Stufe oxydiert, was an der auftretenden Gelbfärbung $z u$ erkennen ist. Diesem Übelstande kann jedoch leicht durch Hinzufügen einiger Tropfen Wasserstoffsuperoxyd abgeholfen werden.

Aus $5 \mathrm{~g}$ Ceronitrat und $2 \mathrm{~g}$ Natriumnitrat erhält man ungefähr 5 g des Doppelnitrats. Dasselbe besteht aus weilsen, feinnadeligen 
Kristallen, die ziemlich hygroskopisch sind. Das Cero-Natriumnitrat ist mit dem entsprechenden Natriumsalze isomorph, denn es kristallisiert aus seinen konzentrierten Lösungen beim Impfen mit letzterem Salze. Auch hier liefs sich das Wasser beim Erhitzen bis auf $150^{\circ}$ nicht vollständig aus dem. Salze entfernen.

$0.2546 \mathrm{~g}$ Subst.: $0.0850 \mathrm{~g} \mathrm{CeO}_{2} ; 0.0716 \mathrm{~g}$ Subst.: $17.6 \mathrm{ccm}$ NO bei $721.2 \mathrm{~mm}, 22^{\circ} ; 0.3415 \mathrm{~g}$ Subst.: $0.0964 \mathrm{~g} \mathrm{Na}_{2} \mathrm{SO}_{4}$.

Für $\left[\mathrm{Ce}\left(\mathrm{NO}_{3}\right)_{5}\right] \mathrm{Na}_{2} \cdot \mathrm{H}_{2} \mathrm{O}$ berechnet $\mathrm{CeO}_{2} 33.49$ NO $29.17 \quad \mathrm{Na} 8.94$ gefunden $\mathrm{CeO}_{2} 33.39$ NO $28.97 \quad \mathrm{Na} 9.13$

Die Ermittelung der Dichte bei $0^{\circ}$ ergab $d_{40}^{00}=2.65$. Das Molekularvolumen ist somit 194.0.

\section{Cero-Rubidiumnitrat $\left[\mathrm{Ce}\left(\mathrm{NO}_{3}\right)_{5}\right] \mathrm{Rb}_{2} \cdot 4 \mathrm{H}_{2} \mathrm{O}$.}

Das Cero-Rubidiumnitrat wurde nach dem gleichen Verfahren wie das Lanthansalz, mit dem es isomorph ist, dargestellt. Die Bildung eines sauren Salzes konnte nicht beobachtet werden. Aus $10 \mathrm{~g}$ Ceronitrat und $7 \mathrm{~g}$ Rubidiumnitrat werden $12 \mathrm{~g}$ des Doppelnitrats erhalten. Dasselbe stellt kleine weifse, monokline Kristalle dar, die ziemlich hygroskopisch sind und bei $70^{\circ}$ in ihrem Kristallwasser schmelzen. Die anhaftende Salpetersäure kann aus dem Salze auch beim tagelangen Verweilen über Kaliumhydroxyd nicht vollständig entfernt werden. Bei $60^{\circ}$ getrocknet, verliert das Doppelnitrat sämtliches Wasser.

02541 g Subst.: $0.0635 \mathrm{~g} \mathrm{CeO}_{2} ; 0.0492 \mathrm{~g}$ Subst.: $9.2 \mathrm{ccm}$ NO bei $723 \mathrm{~mm}, 23^{\circ} ; 0.4334 \mathrm{~g}$ Subst.: $0.1676 \mathrm{~g} \mathrm{Rb}_{2} \mathrm{SO}_{4}$; $0.3806 \mathrm{~g}$ Subst. verloren bei $60^{\circ} 0.0398 \mathrm{~g}$.

Für $\left[\mathrm{Ce}\left(\mathrm{NO}_{3}\right)_{5}\right] \mathrm{Rb}_{2}, 4 \mathrm{H}_{2} \mathrm{O}$ ber. $\mathrm{CeO}_{2} 24.84 \mathrm{NO} 21.64 \mathrm{Rb} 24.65 \mathrm{H}_{2} \mathrm{O} 10.39$ gef. $\mathrm{CeO}_{2} 24.90 \mathrm{NO} 22.03 \mathrm{Rb} 24.75 \mathrm{H}_{2} \mathrm{O} 10.45$

Die Bestimmung der Dichte bei $0^{0}$ ergab $d_{4^{\circ}}^{00}=2.497$. Das Molekularvolumen ist daher 277.6.

\section{Cero-Thallonitrat $\left[\mathrm{Ce}\left(\mathrm{NO}_{3}\right)_{5}\right] \mathrm{Tl}_{2} \cdot 4 \mathrm{H}_{2} \mathrm{O}$.}

Das Cero-Thallonitrat lärst sich nach der gleichen Methode wie das isomorphe Lanthan-Thallonitrat darstellen. $5 \mathrm{~g}$ Ceronitrat und $3.1 \mathrm{~g}$ Thallonitrat geben $7 \mathrm{~g}$ des Doppelsalzes. Dasselbe kristallisiert in weifsen, glänzenden; zu Drusen vereinigten Kristallen. Es ist sehr hygroskopisch und wird durch Wasser sofort zersetzt. Sein 
Schmelzpunkt liegt bei $64.5^{\circ}$. Seine wässerige Lösung reagiert neutral.

$0.3346 \mathrm{~g}$ Subst.: $0.0620 \mathrm{~g} \mathrm{CeO}_{2} ; 0.5100 \mathrm{~g}$ Subst.: $22 \mathrm{ccm}$ $\mathrm{KMnO}_{4}$-Lösung, $1 \mathrm{ccm} \mathrm{KMnO}_{4}$-Lösung $=0.000797 \mathrm{~g} 0 ; 0.0820 \mathrm{~g}$ Subst.: $11.3 \mathrm{ccm}$ NO bei $725 \mathrm{~mm}, 23^{\circ} ; 0.3836 \mathrm{~g}$ verloren bei $60^{\circ}$ $0.0306 \mathrm{~g}$.

Für $\left.\left[\mathrm{Ce}_{(}^{\prime} \mathrm{NO}_{3}\right)_{5}\right] \mathrm{Tl}_{2} \cdot 4 \mathrm{H}_{2} \mathrm{O}$ ber. $\mathrm{CeO}_{2} 18.51$ Tl $43.85 \mathrm{NO} 16.12 \mathrm{H}_{2} 07.74$ gef. $\mathrm{CeO}_{2} 18.52$ Tl 43.83 NO $16.28 \mathrm{H}_{2} 07.97$

Die Dichtenbestimmung bei $0^{0}$ ergab $d_{4^{0}}^{00}=3.326$. Das Molekularvolumen ist daher 279.7 .

Praseodym-Rubidiumnitrat $\left[\operatorname{Pr}\left(\mathrm{NO}_{3}\right)_{5}\right] \mathrm{Rb}_{2} .4 \mathrm{H}_{2} \mathrm{O}$.

$2 \mathrm{~g}$ Praseodymperoxyd und $3.4 \mathrm{~g}$ Rubidiumnitrat werden in Salpetersäure gelöst. Es ist zweckmäfsig hierbei einige Tropfen Wasserstoffsuperoxyd zuzusetzen, wodurch das Praseodymperoxyd reduziert und dann durch die Säure leichter gelöst wird. Die grüne Lösung wird nachher bis auf ungefähr $8 \mathrm{ccm}$ eingedampft und nach dem Erkalten mit einer Spur des isomorphen Cerosalzes geimpft, worauf das Doppelnitrat in lauchgrünen, monoklinen, glänzenden Kristallen in einer Ausbeute von $4.5 \mathrm{~g}$ erhalten wird. Durch Trocknen über Kaliumhydroxyd kann es von der anhaftenden Salpetersäure befreit werden. Das Praseodym-Rubidiumnitrat ist sehr hygroskopisch und schmilzt bei $63.5^{\circ}$ in seinem Kristallwasser. Seine wässerige Lösung reagiert neutral.

$0.2292 \mathrm{~g}$ Subst.: $0.0541 \mathrm{~g} \operatorname{Pr}_{2} \mathrm{O}_{3} ; 0.0422 \mathrm{~g}$ Subst.: $7.8 \mathrm{ccm}$ NO bei $720 \mathrm{~mm}, 24^{0} ; 0.2292 \mathrm{~g}$ Subst.: $0.0890 \mathrm{~g} \mathrm{Rb}_{2} \mathrm{SO}_{4}$; $0.3111 \mathrm{~g}$ Subst.: $0.0335 \mathrm{~g}$ Verlust bei $60^{\circ}$.

Für $\left[\operatorname{Pr}\left(\mathrm{NO}_{3}\right)_{5}\right] \mathrm{Rb}_{2}, 4 \mathrm{H}_{2} \mathrm{O}$ ber. $\mathrm{Pr}_{2} \mathrm{O}_{3} 23.73 \mathrm{NO} 21.63 \mathrm{Rb} 24.64 \mathrm{H}_{2} \mathrm{O} 10.38$ gef. $\mathrm{Pr}_{2} \mathrm{O}_{3} 23.62 \mathrm{NO} 21.61 \mathrm{Rb} 24.86 \mathrm{H}_{2} \mathrm{O} 10.76$

Die Dichtenbestimmung bei $0^{0}$ ergab $d_{4^{\circ}}^{0^{0}}=2.50^{\circ}$. Das Molekularvolumen ist somit 277.4.

\section{Neodym-Rubidiumnitrat $\left[\mathrm{Nd}\left(\mathrm{NO}_{3}\right)_{5}\right] \mathrm{Rb}_{2} .4 \mathrm{H}_{2} \mathrm{O}$.}

Die Darstellung des Neodym-Rubidiumnitrats ist der des entsprechenden Lanthansalzes vollkommen gleich. Um ein einheitliches Produkt zu erhalten, ist es notwendig, die Kristallisation bei möglichst niedriger Temperatur vorzunehmen. Aus $2 \mathrm{~g}$ Neodymoxyd und $3.8 \mathrm{~g}$ Rubidiumnitrat wurden $4 \mathrm{~g}$ des Doppelnitrats erhalten. 
Das Neodym-Rubidiumnitrat kristallisiert in kleinen bellrot. violetten, zu Drusen vereinigten Tafeln. Sein Schmelzpunkt liegt bei $47^{\circ}$. Es ist sehr hygroskopisch und seine wässerige Lösung reagiert neutral.

0.2552 g Subst.: $0.0614 \mathrm{~g} \mathrm{Nd}_{2} \mathrm{O}_{3} ; 0.1000$ g Subst.: $18.6 \mathrm{ccm} \mathrm{NO}$ bei $712 \mathrm{~mm}, 22^{0} ; 0.2794 \mathrm{~g}$ Subst.: $0.107 \mathrm{~s} \mathrm{~g} \mathrm{Rb}_{2} \mathrm{SO}_{4} ; 0.3112 \mathrm{~g}$ Subst. verloren bei $60^{\circ} 0.0322 \mathrm{~g}$.

Für $\left[\mathrm{Nd}\left(\mathrm{NO}_{3}\right)_{5}\right] \mathrm{Rb}_{2} .4 \mathrm{H}_{2} \mathrm{O}$ ber. $\mathrm{Nd}_{2} \mathrm{O}_{3} 24.13 \mathrm{Rb} 24.50 \mathrm{NO} 21.51 \mathrm{H}_{2} \mathrm{O} 10.33$ gef. $\mathrm{Nd}_{2} \mathrm{O}_{3} 24.05 \mathrm{Rb} 24.69 \mathrm{NO} 21.65 \mathrm{H}_{2} \mathrm{O} 10.34$

Die Bestimmung der Dichte bei $0^{0}$ ergab $d_{4^{0}}^{0^{0}}=2.56$. Daher ist das Molekularvolumen 272.3.

Zïrich, Chemisches Universitätslaboratorium, Oktober 1910.

Bei der Redaktion eingegangen am 20. Oktober 1910. 